\title{
Rational Continuity: \\ Parametric, Geometric, and Frenet Frame Continuity of Rational Curves
}

\author{
MICHAEL E. HOHMEYER and BRIAN A. BARSKY \\ University of California at Berkeley
}

\begin{abstract}
The parametric, geometric, or Frenet frame continuity of a rational curve has often been ensured by requiring the homogeneous polynomial curve associated with the rational curve to possess either parametric, geometric, or Frenet frame continuity, respectively. In this paper, we show that this approach is overly restrictive and derive the constraints on the associated homogeneous curve that are both necessary and sufficient to ensure that the rational curve is either parametrically, geometrically, or Frenet frame continuous.
\end{abstract}

Categories and Subject Descriptors: I.3.5 [Computer Graphics]: Computational Geometry and Object Modeling-curve, surface, solid, and object representations; J.6 [Computer Applications]: Computer-Aided Engineering-computer-aided design (CAD)

General Terms: Algorithms, Design

Additional Key Words and Phrases: Beta-constraints, Beta-splines, Bézier curves, B-splines, computer-aided geometric design, continuity, curves and surfaces, Frenet frame, Frenet-frame continuity, geometric continuity, jet spaces, NURBs, parametric continuity, rational B-splines, rational continuity, rational splines, reparameterization

\section{INTRODUCTION}

Rational parametric curves have been receiving considerable attention in the areas of computer graphics and geometric modeling. This is due in part to the fact that most common primitives such as conics $[38,39,41-43]$ as well as freeform geometry can be represented by this single formulation. The rational curve has manifested itself in various forms, including the nonuniform rational Bspline (or the NURB as it is popularly called) $[43,47,48]$, the rational Bézier curve $[18,40]$, and the rational Beta-spline $[2,4,10,21,22,31]$. Any parametric polynomial curve can be expressed as a rational curve, and most polynomial splines and curves have rational extensions.

Since a single rational function usually does not have enough freedom to represent a given curve, several rational segments are used instead. To generate a curve of satisfactory smoothness, the segments must connect with some amount

This work was supported in part by National Science Foundation Presidential Young Investigator Award CCR-8451997.

Authors' address: Computer Science Division, Department of Electrical Engineering and Computer Sciences, University of California at Berkeley, Berkeley, CA 94720.

Permission to copy without fee all or part of this material is granted provided that the copies are not made or distributed for direct commercial advantage, the ACM copyright notice and the title of the publication and its date appear, and notice is given that copying is by permission of the Association for Computing Machinery. To copy otherwise, or to republish, requires a fee and/or specific permission.

(C) 1990 ACM 0730-0301/89/1000-0335 $\$ 01.50$ 
of continuity. Thus, the use of rational curves, independent of the particular variety, creates a common problem: that of connecting rational segments to form piecewise rational curves that are smooth.

In this paper, we concern ourselves with three kinds of continuity for parametric curves: parametric continuity, geometric continuity, and Frenet frame continuity. A curve is said to possess parametric continuity $C^{n}$ if each of the components of the curve is $C^{n}$. It has been shown that a curve possesses geometric continuity $G^{n}$ if and only if each of the components of the curve satisfies a set of constraints called the Beta-constraints [5-7, 14]. A curve possesses Frenet frame continuity if and only if it satisfies a similar set of componentwise constraints [16]. To obtain rational curves with parametric or geometric continuity, parametric continuity constraints or Beta-constraints have generally been applied, not to the components of the rational curve, but to the components of the curve represented in homogeneous coordinates. Alternatively, if the homogeneous curve is not required to be smooth, ad hoc methods have been used to show that the rational curve will be smooth $[2,18,21,22,31,47]$. It is true that if the homogeneous curve satisfies the relevant continuity constraints then the rational curve will have the corresponding continuity. However, it is not true that the homogeneous curve must satisfy these constraints to have this continuity. That is, the constraints are sufficient but not necessary. In this paper, we derive the constraints that are both necessary and sufficient to ensure continuity for rational curves. $^{1}$

\section{RATIONAL CURVES}

A rational function is a scalar function, $r: \mathbb{R} \rightarrow \mathbb{R}$ that can be expressed as

$$
r(u)=\frac{f(u)}{g(u)}
$$

where $f$ and $g$ are polynomials in $u$. Clearly, all polynomial functions are rational functions. We restrict our use of the word polynomial as follows: A rational function is not a polynomial function unless its denominator divides its numerator. Such functions are sometimes referred to as integral functions to distinguish them from rational functions. On the other hand, for our purposes, polynomials include piecewise polynomials. A curve is simply a vector-valued function q: $\mathbb{R} \rightarrow \mathbb{R}^{d}$. A polynomial curve is a vector-valued function, each component of which is a polynomial, and a rational curve is a vector-valued function, each component of which is a rational function. There are at least two ways to visualize the mathematics of a rational curve. The curve $q$ can be thought of as a vector-valued function, each component of which is a rational function, or q can be thought of as the composition of a vector-valued polynomial function $\mathbf{Q}: \mathbb{R} \rightarrow \mathbb{R}^{d+1}$ with a projection function that takes $\left(x_{1}, \ldots, x_{d}, x_{d+1}\right)$ to $\left(x_{1} / x_{d+1}, \ldots, x_{d} / x_{d+1}\right)$.

In the first view, one would write

$$
\mathbf{q}(u)=\left(q_{1}(u), q_{2}(u), \ldots, q_{d}(u)\right)
$$

\footnotetext{
${ }^{1}$ After reading an earlier version of this manuscript containing more computational proofs not based on $n$-jets, Goldman and Micchelli [23] independently found some similar proofs for many of these results.
}

ACM Transactions on Graphics, Vol. 8, No. 4, October 1989. 
where each component function, $q_{i}$, is the rational function

$$
q_{i}(u)=\frac{Q_{i}(u)}{Q_{d+1}(u)}
$$

and the $Q_{i}$ s are all polynomial functions. Note that the denominator of each component function is the same polynomial $Q_{d+1}$. In general, this is not a severe restriction since, if the denominator of each $q_{i}$ were $g_{i}$, one could multiply the numerator and the denominator of $q_{i}$ by $l(u) / g_{i}(u)$, where $l$ is the least common multiple of $\left\{g_{1}, \ldots, g_{d}\right\}$, to obtain a satisfactory set of $Q_{i} s$.

In the second view, one might think of the same curve as a polynomial curve whose range is the homogeneous coordinate system of dimension $d+1$. In this case, the division by $Q_{d+1}$ takes place implicitly. The rational curve $\mathbf{q}$, discussed above, would be represented in this scheme by the polynomial curve $\mathbf{Q}$, where

$$
\mathbf{Q}(u)=\left(Q_{1}(u), Q_{2}(u), \ldots, Q_{d}(u), Q_{d+1}(u)\right) .
$$

We refer to $\mathbf{Q}$ as the "homogeneous curve associated with $\mathbf{q}$ " (even though it is not unique) and $\mathbf{q}$ as the "projection of $\mathbf{Q}$ " (which is unique). Also, we use italics to indicate scalar-valued functions, such as $q_{i}$ or $Q_{i}$; boldface lowercase to indicate vector-valued rational curves, such as $\mathbf{q}$, whose range is $\mathbb{R}^{d}$, the space in which the curve lies; and boldface uppercase to indicate the associated homogeneous polynomial curve, such as $\mathbf{Q}$. This second view of rational curves and some of the associated notation have been presented in earlier works by Roberts [44] and Cohen and Lee [12].

To illustrate more concretely, consider a curve formulation such as the rational Bézier curve, the rational B-spline curve, or the rational Beta-spline curve. Each is a function $\mathbf{q}: \mathbb{R} \rightarrow \mathbb{R}^{d}$ that can be expressed as

$$
\mathbf{q}(u)=\frac{\sum_{i=0}^{m} w_{i} \mathbf{V}_{i} B_{i}(u)}{\sum_{j=0}^{m} w_{j} B_{j}(u)}
$$

where $\mathbf{V}_{i}$ are the control vertices, $B_{i}(u)$ are the basis functions, and $w_{i}$ are the weights for $i=0, \ldots, m .^{2}$ One can also consider such a curve as a polynomial Bézier curve, B-spline, or Beta-spline curve in a higher dimensional space:

$$
\mathbf{Q}(u)=\sum_{i=0}^{m} \mathbf{W}_{i} B_{i}(u)
$$

where $\mathbf{W}_{i}$ are control vertices in $\mathbb{R}^{d+1}$ whose coordinates are $\mathbf{W}_{i}=\left(w_{i} \mathbf{V}_{i}, w_{i}\right)$ for $i=0, \ldots, m$. Of course, when the $\mathbb{R}^{d}$ coordinates of $\mathbf{q}$ are required, the division must be performed. In Figure 1, we show how one might view an $\mathbb{R}^{2}$ rational curve as an $\mathbb{R}^{2+1}$ polynomial curve. Each component of the $\mathbb{R}^{2+1}$ curve is strictly polynomial, and the points on the $\mathbb{R}^{2}$ curve are obtained by projecting the $\mathbb{R}^{2+1}$ curve onto the $w=1$ plane.

The advantage of this perspective is that algorithms to manipulate rational curves (i.e., evaluation, subdivision, degree elevation, bounding, continuity, etc.) can often be obtained by using the corresponding algorithm for polynomial curves. This yields a large body of information on polynomial curves that is

\footnotetext{
${ }^{2}$ The weights are often restricted to be positive to prevent the denominator from becoming zero and to ensure the convex hull property.
} 
Fig. 1. A 2-D rational curve $q$ and its associated 3-D homogeneous curve $\mathbf{Q}$.

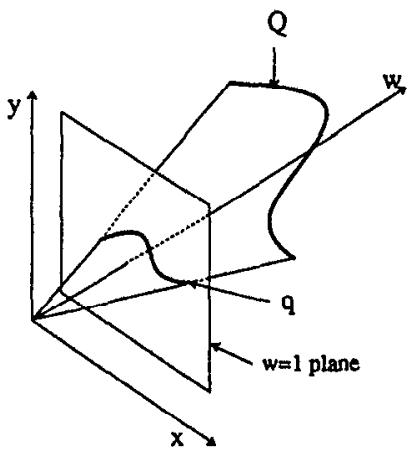

almost always directly applicable to rational curves [18, 40]. For example, to increase the degree of a rational curve $\mathbf{q}$, we increase the degree of the polynomial curve $\mathbf{Q}$ via a well-known algorithm for a polynomial curve. To subdivide $\mathbf{q}$, we subdivide $\mathbf{Q}$, and so on. If we project $\mathbf{Q}$ to obtain $\mathbf{q}$, it will be as if the manipulation had been performed on $\mathbf{q}$.

This method of reducing a problem associated with a rational curve to the analogous problem for its homogeneous counterpart has also been used for the problem of continuity between rational segments. Specifically, parametric and geometric continuity for a rational curve has often been obtained by requiring the associated homogeneous curve to be parametrically [47] or geometrically continuous [2]. In the cases where the homogeneous curve is not required to be smooth, ad hoc methods are used to show that the projected curve will be. Although valid, we show that requiring the homogeneous curve to be as smooth as the projected curve is overly restrictive, and we suggest a more flexible and comprehensive alternative.

\section{PARAMETRIC CONTINUITY}

Conventionally, the "smoothness" of a curve has been measured by testing the continuity of its derivatives. Specifically, a curve $\mathbf{q}$ is said to be $C^{n}$ at $t$ if and only if

$$
\lim _{u \rightarrow t^{+}} \mathbf{q}^{(i)}(u)=\lim _{u \rightarrow t^{-}} \mathbf{q}^{(i)}(u) \quad i=0, \ldots, n
$$

where $u \rightarrow t$ - denotes $u \rightarrow t$ with $u<t$ and where $u \rightarrow t+$ denotes $u \rightarrow t$ with $u>t$. In the above and in the remainder of this paper, we assume that the value of the curve at parameter value $t$ is equal to at least one of the limits.

If we have a homogeneous curve $\mathbf{Q}$ that is $C^{n}$ (with $Q_{d+1}(u) \neq 0$ for all $u$ ), then the projection $\mathbf{q}$ of $\mathbf{Q}$ will also be $C^{n}$. That is, if all the components (the $Q_{i}$ ) of $\mathbf{Q}$ are $C^{n}$ then all the components $Q_{i} / Q_{d+1}$ of $\mathbf{q}$ are $C^{n}$, simply because the quotient of two $C^{n}$ functions is $C^{n}$.

The converse is not true; there are homogeneous curves $\mathbf{Q}$ that are not $C^{n}$ even though their projections $\mathbf{q}$ are $C^{n}$. In Section 10 , we derive the conditions on a homogeneous curve $\mathbf{Q}$ that are exactly equivalent to its projection $\mathbf{q}$ being $C^{n}$. 


\section{GEOMETRIC CONTINUITY}

Recently, it has been suggested that many useful smooth parametric curves do not possess parametric continuity but do have a more relaxed form of continuity called geometric continuity. Two forms of $n$ th-order geometric continuity have been proposed: Beta-constraints [5-7, 13, 14] (sometimes referred to as reparametrization or arc length geometric continuity) and continuity of the Frenet frame (i.e., continuity of generalized curvatures in higher dimensions) $[16,26$, $27,46]$. In this paper we refer to the former concept as geometric continuity and the latter as Frenet frame continuity. ${ }^{3}$

Both $n$ th-order geometric continuity and $n$ th-order Frenet frame continuity are generalizations of first- and second-order geometric continuity. This refers to continuity of the unit tangent and curvature vectors; various forms of this idea were proposed in the computer-aided-geometric-design literature in [1], [3], [17], [19], [20], [34], [37], and [45]. Geometric and Frenet frame continuity have become important topics of research, and recent work has been reported in [8], [9], [11], [21]-[25], [28], and [30].

Geometric continuity is a generalization of parametric continuity that is invariant under reparametrization. Consider objects represented by parametric curves and surfaces. When they are rendered or manufactured, they should show no trace of the manner in which they were parametrized. Thus, measures of continuity should be independent of parametrization. Specifically, a curve is said to be geometrically continuous, denoted $G^{n}$, if and only if there exists a regular ${ }^{4}$ $C^{n}$ parametrization of that curve. Equivalently, a curve is geometrically continuous if and only if its arc length parametrization is parametrically continuous $\left(C^{n}\right)$. Clearly, all regular $C^{n}$ curves are $G^{n}$, but not conversely. Barsky and DeRose introduced the notion of geometric continuity and derived constraints, called Beta-constraints, on the components of $\mathbf{q}$ (not on the components of $\mathbf{Q}$ ) that hold if and only if the curve is geometrically continuous [5-7, 13, 14].

In this section, we discuss the zeroth through the second Beta-constraints. In Section 8 , we discuss the Beta-constraints associated with $G^{n}$ for arbitrary $n$.

The zeroth Beta-constraint is identical to the zeroth parametric continuity constraint; it requires that

$$
\lim _{u \rightarrow t^{+}} \mathbf{q}^{(0)}(u)=\lim _{u \rightarrow t^{-}} \mathbf{q}^{(0)}(u) .
$$

The first Beta-constraint says that a curve $\mathbf{q}$ is $G^{1}$ at $t$ if and only if

$$
\lim _{u \rightarrow t^{+}} \mathbf{q}^{(1)}(u)=\lim _{u \rightarrow t^{-}} \beta_{1} \mathbf{q}^{(1)}(u)
$$

for some $\beta_{1}>0$. That is, if two curve segments join in a way that appears to be $C^{1}$ then their first derivatives need not be identical, but rather one need only be a positive scalar multiple of the other.

\footnotetext{
${ }^{3}$ Although the term geometric continuity was originally coined in the computer graphics and modeling community in [1] and [3] and was used to refer to Beta-constraints in [5], it has also been subsequently used to indicate "Frenet frame continuity" in [11], [15], [16], [26], and [27].

"A parametrization of a curve $q$ is regular if its first derivative vector $q^{(1)}$ is never the zero vector.
} 
The curve $\mathbf{q}$ is $G^{2}$ at $t$ if and only if, in addition to eq. (9),

$$
\lim _{u \rightarrow t^{+}} \mathbf{q}^{(2)}(u)=\lim _{u \rightarrow t^{-}}\left[\beta_{1}^{2} \mathbf{q}^{(2)}(u)+\beta_{2} \mathbf{q}^{(1)}(u)\right]
$$

for some $\beta_{2}$.

The variables $\beta_{1}$ and $\beta_{2}$ are called shape parameters. A curve that is $G^{n}$ will have $n$ shape parameters. Although the shape parameters cannot be obtained from the shape of a curve alone, if we restrict our curves to be polynomial, for example, then varying the shape parameters will affect the shape of the curve, hence, the name $[1,3]$.

It has been shown that if $\mathbf{Q}$, the homogeneous curve associated with $\mathbf{q}$, is $G^{n}$ and $\mathbf{q}$ is regular then $\mathbf{q}$ will be $G^{n},[21,22]$. Just as with parametric continuity, there are homogeneous curves $\mathbf{Q}$ that are not $G^{n}$ even though their projections, $\mathbf{q}$, are $G^{n}$. In Section 9 we generalize the notion of $G^{n}$ to include curves whose projections are geometrically continuous.

\section{FRENET FRAME CONTINUITY}

Frenet frame continuity is based on the continuity of generalized curvatures in higher dimensions. If $\mathbf{q}(u)$ is a curve in $\mathbb{R}^{d}$ that is parametrized by arc length, then its Frenet frame of orthonormal vectors $\mathbf{v}_{1}(u), \ldots, \mathbf{v}_{d}(u)$ and its higher order scalar curvatures are defined recursively by

$$
\begin{gathered}
\mathbf{v}_{1}(u)=\mathbf{q}^{\prime}(u) \\
\kappa_{0}(u)=0 \\
\mathbf{v}_{i+1}(u)=\frac{\mathbf{v}_{i}^{\prime}(u)+\kappa_{i-1}(u) \mathbf{v}_{i-1}(u)}{\kappa_{i}(u)} \quad i=1, \ldots, d-1
\end{gathered}
$$

where $\kappa_{i}(u)$ is chosen so that $\mathbf{v}_{i+1}(u)$ has unit length. In three dimensions, $\kappa_{1}(u)$ and $\kappa_{2}(u)$ are the familiar quantities curvature and torsion, respectively $[15,26$, $27,44]$. A curve $\mathbf{q}(u)$ is said to be Frenet frame continuous of order $n$ (which we will denote $F^{n}$ ) if and only if the Frenet frame vectors and curvatures of its arc length parametrization are continuous.

Frenet frame continuity and geometric continuity agree for orders of continuity 1 and 2. For $n>2, F^{n}$ is a generalization of $G^{n}$. Unlike $G^{n}$, for $n>2$, there are curves that are $F^{n}$ that do not possess a regular $C^{n}$ parametrization. It is important to note that all curves in $\mathbb{R}^{c}$ that are $F^{d}$ will trivially be $F^{i}$ for $i \geq d$. For example, all planar curves that are $F^{2}$ are also $F^{3}, F^{4}, \ldots$, and all curves in $\mathbb{R}^{3}$ that are $F^{3}$ are also $F^{4}, F^{5}, \ldots$ Thus, in $\mathbb{R}^{d}$, Frenet frame continuity only distinguishes among $d+2$ classes of curves: those that are positionally discontinuous, those that are only positionally continuous, those that are $F^{i}$ but not $F^{i+1}, i=$ $1, \ldots, d-1$, and those that are $F^{d}$. 'This contrasts with geometric continuity, which distinguishes between an infinite number of classes of curves.

As with parametrically and geometrically continuous curves, if $\mathbf{Q}$, the homogeneous curve associated with $\mathbf{q}$, is $F^{n}$ and $\mathbf{q}$ is regular then $\mathbf{q}$ will be $F^{n}[22,23]$. There is a subtle difference, however. Just as with geometric continuity, there is a set of scalar values called shape parameters that are determined by the geometry and the parametrization of the curve. These are different shape parameters, 
however, than those involved in the Beta-constraints; in fact, there are more shape parameters for Frenet frame continuity than for geometric continuity. In the case of geometric continuity, the shape parameters are invariant under projection. That is, the shape parameters for $\mathbf{Q}$ are the same as the shape parameters for $\mathbf{q}$. For Frenet frame continuous curves in general, the shape parameters are not invariant under projection. It has been shown in [22] and [23] that no generalization of geometric continuity (such as Frenet frame continuity) can have this invariance property; that is, the shape parameters are invariant if and only if the curve is geometrically continuous. Nevertheless, there exist homogeneous curves $\mathbf{Q}$ that are not $F^{n}$ even though their projections are $F^{n}$. In Section 8, we generalize the notion of $F^{n}$ to include curves whose projections are Frenet frame continuous.

\section{THE NEED FOR MORE GENERAL CONTINUITY}

There exist curves $\mathbf{Q}$, of practical use, that are not $C^{n}$, and in fact are not even $G^{n}$ nor $F^{n}$, but whose projections $\mathbf{q}$ are not only $F^{n}$ and $G^{n}$ but even $C^{n}$. Our aim is to derive componentwise continuity constraints on $\mathbf{Q}$ that will be necessary and sufficient to ensure that $\mathbf{q}$ is either $C^{n}, G^{n}$, or $F^{n}$.

Complete circles are typically represented with rational curves using four quadratic rational segments-either four quadratic rational Bézier curves or equivalently by a quadratic NURB with four segments and double knots [47]. In Figure 2, we have shown this as a projection of a homogeneous curve, $\mathbf{Q} \in \mathbb{R}^{2+1}$, onto the $w=1$ plane, to obtain the rational curve $\mathbf{q} \in \mathbb{R}^{2}$. Note that the homogeneous curve segments are copies of one another rotated 90 degrees about the $w$ axis.

We can see that $\mathbf{Q}$ is not $C^{1}$ at the knots. In fact, it is not even $G^{1}$. On the contrary, $\mathbf{Q}$ has highly visible "kinks" in it. Since the segments of $\mathbf{q}$ lie on the circle, however, their tangents must be in the same direction as the circle tangents. Also, since the segments are congruent, the tangents must have the same magnitude. Thus, $\mathbf{q}$ is $C^{1}$ and $G^{1}$, even though $\mathbf{Q}$ is neither. And, even though the NURB q that represents this curve is $C^{1}$, it must have double knots. This may be surprising because a piecewise polynomial curve of order $k$ and continuity $C^{n}$ can be represented by a B-spline with knot multiplicity $k-n-1$. If the curve under consideration is a B-spline with knot multiplicity greater than $k-n-1$ (but still of continuity $C^{n}$ ), knots can be removed (without changing the curve) until the knot multiplicity is $k-n-1$. In the case mentioned above, $k=3$ and $n=1$, so that one might suspect that $\mathbf{q}$ would only need single knots. $\mathrm{Be}$ reminded, however, that $\mathbf{q}$ is a NURB and not necessarily a B-spline. Apparently, the B-spline multiplicity rule does not apply to NURBs. By viewing a NURB as a projection of a B-spline from $\mathbb{R}^{d+1}$ to $\mathbb{R}^{d}$, we remind ourselves that NURBs are not B-splines and yet are closely related.

One might reasonably ask if it is possible to represent the circle with a sequence of quadratic segments that meet with $C^{1}$ continuity in $\mathbb{R}^{2+1}$, thus dispensing with the need for a more generalized continuity. We will show that if two quadratic segments in $\mathbb{R}^{2+1}$ that project onto this circle meet with $C^{1}$ continuity (in $\mathbb{R}^{2+1}$ ) then they are the same polynomial curve. Also, if a single quadratic segment is used, the parameterization must run from $-\infty$ to $\infty$. 
Fig. 2. A circle represented by a piecewise rational curve.
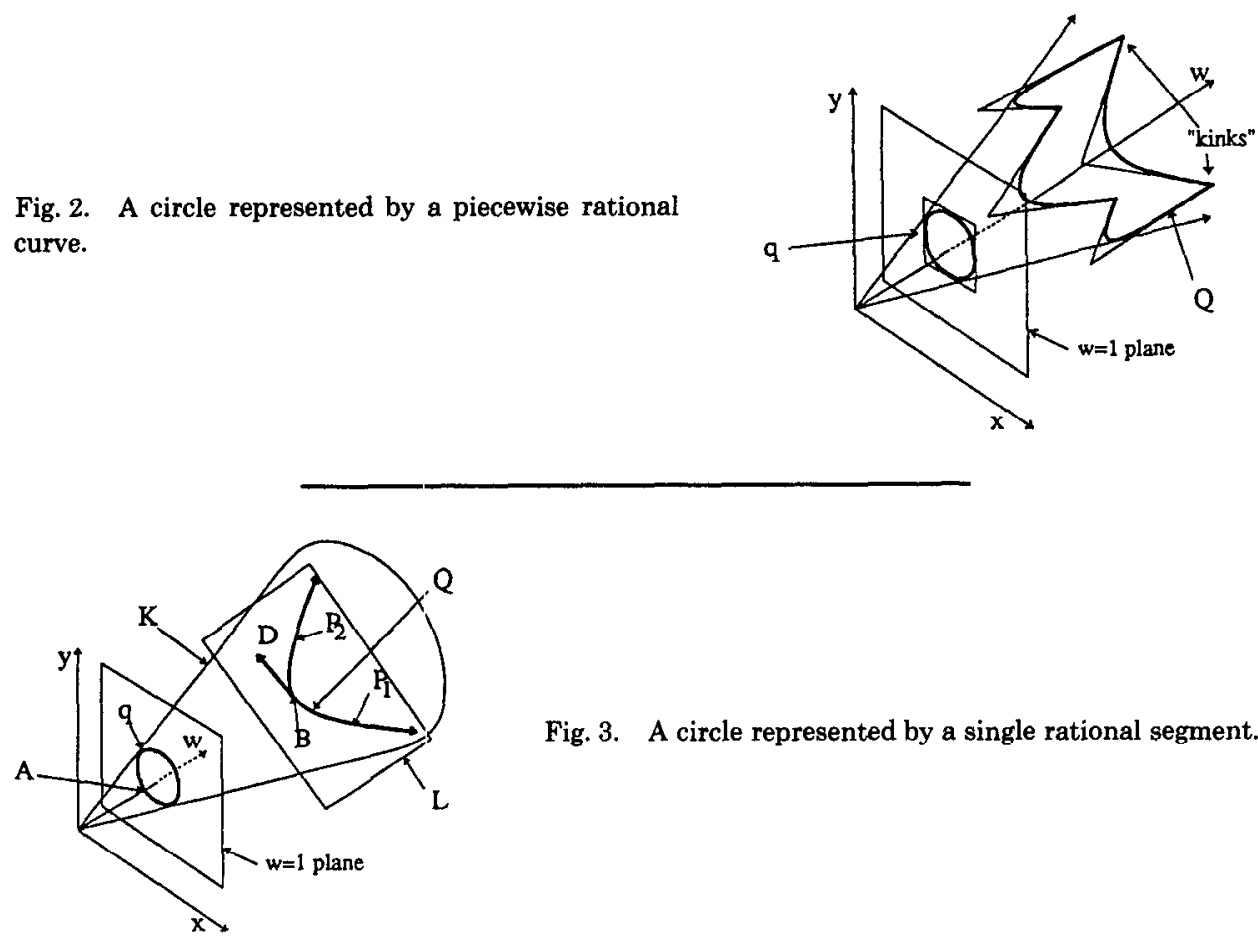

Fig. 3. A circle represented by a single rational segment.

In order for $\mathbf{q}$ to be a circle, $\mathbf{Q}$ must lie on the cone that projects to $\mathbf{q}$. This cone is denoted $K$ in Figure 3. Since all quadratic curves are planar, then, in particular, so is $\mathbf{Q}$. The plane in which $\mathbf{Q}$ lies is denoted $L$ in Figure 3. Thus, $\mathbf{Q}$ must lie in the intersection of $K$ and $L$. Such an intersection will be either an ellipse, an hyperbola, or a parabola. Since $\mathbf{Q}$ must be strictly polynomial, the intersection must be a parabola. As $u \rightarrow-\infty$ or $+\infty$, $\mathbf{Q}$ diverges and $\mathbf{q}$ slowly approaches the point $\mathbf{A}$ in Figure 3.

One might try to obtain a more usable parameterization by composing $\mathbf{Q}$ from two quadratic segments $\mathbf{P}_{1}$ and $\mathbf{P}_{2}$ that meet with $C^{1}$ continuity. $\mathbf{P}_{1}$ and $\mathbf{P}_{2}$ have to be the same polynomial (up to a linear change of parameter) for the following reason: At the point $\mathbf{B}$ in Figure 3, where $\mathbf{P}_{1}$ and $\mathbf{P}_{2}$ meet on the cone, $\mathbf{P}_{1}$ and $\mathbf{P}_{2}$ must share a first derivative vector, $\mathbf{D}$. Each of $\mathbf{P}_{1}$ and $\mathbf{P}_{2}$ must lie in one of the infinitely many planes defined by $\mathbf{D}$ and $\mathbf{B}$. However, only one of these planes has an intersection with the cone that is a parabola. Thus, both $\mathbf{P}_{1}$ and $\mathbf{P}_{2}$ must lie in the same plane and on the same intersection parabola, and they must be the same parabola. Consequently, if we insist that our curves be quadratic, and a parameterization from $-\infty$ to $+\infty$ is not acceptable, then we must construct $\mathbf{Q}$ from several segments that do not meet with $C^{1}$ continuity.

This example demonstrates that we cannot apply parametric continuity constraints or Beta-constraints to $\mathbf{Q}$ to test the continuity of $\mathbf{q}$. That is, parametric continuity constraints and Beta-constraints are too restrictive to be applied to the homogeneous counterpart of a curve. However, the continuity constraints that we will derive admit the homogeneous curve $\mathbf{Q}$ in Figure 2. Since it is ACM Transactions on Graphics, Vol. 8, No. 4, October 1989. 
necessary to be able to represent circles in a CAD application, we believe that there are important curves that satisfy our new continuity constraints but do not satisfy either the parametric continuity constraints or the Beta-constraints.

\section{PARAMETRIC CONTINUITY OF QUOTIENTS}

In the above, we have seen a homogeneous curve $\mathbf{Q}$ that is not $C^{1}$ but whose projection $\mathbf{q}$ is $C^{1}$. If we examine a single component $Q_{i} / Q_{d+1}$ of $\mathbf{q}$, we see that the quotient is $C^{1}$ even though neither $Q_{i}$ nor $Q_{d+1}$ is. The problem, in general, is that it is not necessary for each of two scalar functions, $Q_{i}$ and $Q_{d+1}$, to be $C^{n}$ in order that $Q_{i} / Q_{d+1}$ be $C^{n}$.

What constraints must we impose on two scalar functions, $Q_{i}$ and $Q_{d+1}$, in order that the quotient $Q_{i} / Q_{d+1}$ is $C^{n}$ ? Later in this paper, we present a general method for deriving these continuity constraints, but first we present a more instructive approach. Consider a scalar function $r(u)$ that is composed of two $C^{n}$ functions, $p(u)$ for $u \leq t$ and $q(u)$ for $u>t$, where $p(u)=P_{i}(u) / P_{d+1}(u)$ and $q(u)=Q_{i}(u) / Q_{d+1}(u)$, as is the case with piecewise rational functions. Clearly, $r$ will be $C^{n}$ if and only if

$$
\left.\left(\frac{Q_{i}(u)}{Q_{d+1}(u)}\right)^{(j)}\right|_{u=t}=\left.\left(\frac{P_{i}(u)}{P_{d+1}(u)}\right)^{(j)}\right|_{u=t} \quad j=0, \ldots, n .
$$

\subsection{Positional Continuity}

For positional continuity $(n=0)$, this reduces to

$$
\frac{Q_{i}(t)}{Q_{d+1}(t)}=\frac{P_{i}(t)}{P_{d+1}(t)} .
$$

If we cross multiply and introduce a parameter $\alpha_{0}$, then

$$
\frac{Q_{i}(t)}{P_{i}(t)}=\frac{Q_{d+1}(t)}{P_{d+1}(t)}=\alpha_{0} .
$$

Note that $\alpha_{0} \neq 0$ since neither $Q_{d+1}$ nor $P_{d+1}$ is ever zero. We can separate the constraints on $P$ and $Q$ into

$$
Q_{i}(t)=\alpha_{0} P_{i}(t)
$$

and

$$
Q_{d+1}(t)=\alpha_{0} P_{d+1}(t)
$$

Written in this form, the constraint (17) on the $i$ th components of $P$ and $Q$ is the same as the constraint (18) on the $d+1$ st components of $P$ and $Q$, which is slightly surprising considering their asymmetric roles as numerator and denominator. However, this allows us to make the following observation: Consider a rational curve $\mathbf{r}(u)$ comprising two curves $\mathbf{p}(u)$ for $u \leq t$ and $\mathbf{q}(u)$ for $u>t$, where $\mathbf{p}, \mathbf{q}: \mathbb{R} \rightarrow \mathbb{R}^{d}$. The components of $\mathbf{p}(u)$ are $P_{i}(u) / P_{d+1}(u)$ and similarly for $\mathbf{q}(u)$. If $\mathbf{r}$ is $C^{0}$ at $t$, then the $i$ th component must be $C^{0}$, and equation (17) must be satisfied for $i=1, \ldots, d$. Since $\alpha_{0}$ is determined by the $d+1^{\text {st }}$ component, then each component must satisfy equation (17) for the same value of $\alpha_{0}$. Thus, 
Fig. 4. For the rational curve $\mathbf{r}$ to be $C^{0}, \mathbf{P}(t)$ and $\mathbf{Q}(t)$ need only be scalar multiples of one another.

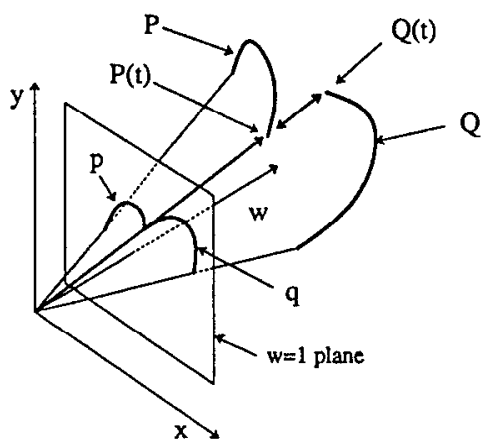

in each dimension $i, i=1, \ldots, d+1$,

$$
Q_{i}(t)=\alpha_{0} P_{i}(t) \text {. }
$$

Consider the equivalent restrictions on the homogeneous curve $\mathbf{R}(u)$ associated with $\mathbf{r}(u)$; they would be

$$
\mathbf{Q}(t)=\alpha_{0} \mathbf{P}(t) .
$$

In Figure 4, we show why this must be the case. Two homogeneous points represent the same projected point if and only if they are scalar multiples of each other. The geometric interpretation of $\alpha_{0}$ then is that $\alpha_{0}$ is the ratio of $\mathbf{Q}(t)$ to $\mathbf{P}(t)$.

\subsection{First Derivative Continuity}

Consider now the case when $n=1$. In addition to eqs. (17) and (18),

$$
\left.\left(\frac{Q_{i}(u)}{Q_{d+1}(u)}\right)^{(1)}\right|_{u=t}=\left.\left(\frac{P_{i}(u)}{P_{d+1}(u)}\right)^{(1)}\right|_{u=t} .
$$

Differentiation yields

$$
\frac{Q_{i}^{\prime}(t) Q_{d+1}(t)-Q_{i}(t) Q_{d+1}^{\prime}(t)}{Q_{d+1}^{2}(t)}=\frac{P_{i}^{\prime}(t) P_{d+1}(t)-P_{i}(t) P_{d+1}^{\prime}(t)}{P_{d+1}^{2}(t)} .
$$

By eqs. (17) and (18),

$$
\frac{Q_{i}^{\prime}(t) \alpha_{0} P_{d+1}(t)-a_{0} P_{i}(t) Q_{d+1}^{\prime}(t)}{\alpha_{0}^{2} P_{d+1}^{2}(t)}=\frac{P_{i}^{\prime}(t) P_{d+1}(t)-P_{i}(t) P_{d+1}^{\prime}(t)}{P_{d+1}^{2}(t)} .
$$

By cross multiplying,

$$
Q_{i}^{\prime}(t) P_{d+1}(t)-P_{i}(t) Q_{d+1}^{\prime}(t)=\alpha_{0} P_{i}^{\prime}(t) P_{d+1}(t)-\alpha_{0} P_{i}(t) P_{d+1}^{\prime}(t) .
$$

Reorganization of terms yields

$$
\left(\alpha_{0} P_{d+1}^{\prime}(t)-Q_{d+1}^{\prime}(t)\right) P_{i}(t)=\left(\alpha_{0} P_{i}^{\prime}(t)-Q_{i}^{\prime}(t)\right) P_{d+1}(t)
$$

If we divide and introduce a parameter $\alpha_{1}$, then

$$
\frac{\alpha_{0} P_{d+1}^{\prime}(t)-Q_{d+1}^{\prime}(t)}{P_{d+1}(t)}=\frac{\alpha_{0} P_{i}^{\prime}(t)-Q_{i}^{\prime}(t)}{P_{i}(t)}=-\alpha_{1} .
$$

ACM Transactions on Graphics, Vol. \&, No. 4, October 1989. 


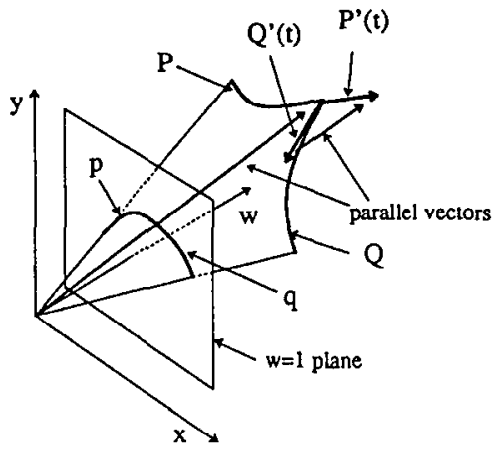

Fig. 5. If $\mathbf{r}$ is $C^{1}$, the first derivatives can differ by a vector parallel to the position vector.

We can separate the constraints on the $i$ th and the $d+1^{\text {st }}$ components of $\mathbf{P}$ and $\mathbf{Q}$ into

$$
Q_{i}^{\prime}(t)=\alpha_{0} P_{i}^{\prime}(t)+\alpha_{1} P_{i}(t)
$$

and

$$
Q_{d+1}^{\prime}(t)=\alpha_{0} P_{d+1}^{\prime}(t)+\alpha_{1} P_{d+1}(t) .
$$

Again, the same constraints are imposed on the $i$ th component as on the $d+1^{\text {st }}$. As before, consider the equivalent constraints on $\mathbf{P}$ and $\mathbf{Q}$, the homogeneous curves associated with $\mathbf{p}$ and $\mathbf{q}$, respectively. By similar reasoning, the restrictions become

$$
\mathbf{Q}^{\prime}(t)=\alpha_{0} \mathbf{P}^{\prime}(t)+\alpha_{1} \mathbf{P}(t) .
$$

We have depicted this constraint in Figure 5 . It should be intuitively clear that if the first derivative of $\mathbf{R}(u)$ has a discontinuity that is along the position vector $\mathbf{P}(t)$ then this discontinuity is not apparent in $\mathbf{r}(u)$. We can attribute the following geometric significance to $\alpha_{1}$ : It is the amount of the position vector that just compensates for the discrepancy in the first derivative of the curve.

We could continue in this manner, developing the continuity constraints for higher degrees of continuity one by one, but the amount of algebra grows unmanageably. We will develop some useful results that will help us to construct the continuity constraints for an arbitrary degree, $n$.

\section{JET SPACES}

We use a special case of jet spaces as described in [29] and [33]. Let $f$ be a univariate function. The $n$-jet of $f$ is defined by

$$
j^{n} f(u)=\left(f^{(0)}(u), f^{(1)}(u), f^{(2)}(u), \ldots, f^{(n-1)}(u), f^{(n)}(u)\right)
$$

or, equivalently,

$$
j_{i}^{n} f(u)=f^{(i)}(u)
$$

If the space of functions $f$ is denoted by $F$, then the space of $n$-jets associated with $F$ will be denoted as $J^{n}(F)$. Just as with the function itself, one can consider the $n$-jet evaluated at a particular parameter value, or one can consider the $n$-jet over the entire range of parameter values. If one considers the $n$-jet over the 
entire range of parameter values, then the $n$-jet completely determines the function. On the other hand, if one considers the $n$-jet at a particular parameter value then several different functions may share the same $n$-jet at the same parameter value. Note that it is possible to construct arbitrary $n$-jet functions $\alpha: \mathbb{R} \rightarrow J^{n}(F)$. Such functions may not be the $n$-jet of any function. That is, there might not exist an $f \in F$ so that $j^{n} f(t)=\alpha(t)$ for all $t$. For example, $\alpha(t)=$ $(t,-1) \in J^{1}(F)$. If $j^{n} f(t)=\alpha(t)$, then we would have $f(t)=t$ and $f^{\prime}(t)=-1$ simultaneously. Nevertheless, there is no reason we cannot consider such $n$-jet functions.

If an operation between two functions $f$ and $g$ (e.g., addition, multiplication, or composition) yields a function $h$ whose first $n+1$ derivatives depend only on the first $n+1$ derivatives of $f$ and $g$, then we can define a corresponding operation between the $n$-jet of $f$ and the $n$-jet of $g$. The result of the operation will be the $n$-jet of $h$. If the functions form a field under addition and multiplication of functions, then we can define these operations on the $n$-jets of the functions and form another field. Let us describe operations of addition and multiplication of $n$-jets in terms of the coordinates of the $n$-jets.

The $n$-jet of the sum of two functions is the sum of the $n$-jets of the two functions

$$
j^{n}(f+g)=j^{n} f+j^{n} g .
$$

We can derive the explicit formula for the coordinates of the sum $n$-jet in terms of the coordinates of the addend $n$-jets.

$$
\left(j^{n} f+j^{n} g\right)_{i}=j_{i}^{n}(f+g)=(f+g)^{(i)}=f^{(i)}+g^{(i)}=j_{i}^{n} f+j_{i}^{n} g .
$$

Clearly, the zero element in the field is the $n$-jet of the zero function. Its coordinates are

$$
j^{n} 0=(0,0,0, \ldots, 0,0)=0 .
$$

The product between $n$-jets is defined so that the $n$-jet of a product of two functions is the product of the $n$-jets.

$$
j^{n}(f g)=j^{n} f * j^{n} g .
$$

In this paper we use the symbol $*$ exclusively to indicate the product of two $n$-jets. Again, we can derive the explicit formula for the coordinates of the product $n$-jet in terms of the coordinates of factor $n$-jets:

$$
\left(j^{n} f * j^{n} g\right)_{i}=j_{i}^{n}(f g)=(f g)^{(i)} .
$$

We can expand this via the Leibnitz rule to yield

$$
\left(j^{n} f * j^{n} g\right)_{i}=\sum_{j=0}^{i} f^{(j)} g^{(j-i)}\left(\begin{array}{l}
i \\
j
\end{array}\right)=\sum_{j=0}^{i} j_{i}^{n} f j_{j-i}^{n} g\left(\begin{array}{l}
i \\
j
\end{array}\right) .
$$

In eq. (37) one could interchange the positions of $j_{j}^{n} f$ and $j_{j-i}^{n} g$ in the summation without affecting the result. Thus, the multiplication of $n$-jets is commutative:

$$
\alpha * \beta=\beta * \alpha \text {. }
$$

ACM Transactions on Graphics, Vol. \&, No. 4, October 1989. 
The identity element in the resulting field is

$$
j^{n} 1=(1,0,0, \ldots, 0) \text {. }
$$

Also, the reciprocal of the $n$-jet of $f$ is the $n$-jet of the reciprocal of $f$ :

$$
j^{n} 1=j^{n} \frac{f}{f}=j^{n} f * j^{n} \frac{1}{f}
$$

We denote this

$$
\frac{1}{j^{n} f}=j^{n} \frac{1}{f}
$$

Reciprocals follow the rule:

$$
\sum_{j=0}^{i}\left(\frac{1}{f}\right)^{(i-j)} f^{(j)}\left(\begin{array}{l}
i \\
j
\end{array}\right)=\left(\frac{1}{j^{n} f} * j^{n} f\right)_{i}=j_{i}^{n} 1=\delta_{i 0} .
$$

The composition, denoted $\circ$, of $n$-jets is defined so that the $n$-jet of the composition of two functions is the composition of the $n$-jets of the two functions,

$$
j^{n}(f \circ g)=\left.j^{n} f\right|_{g} \circ j^{n} g .
$$

Note that composition of $n$-jets is, in general, not commutative since the composition of functions is not commutative. The explicit formula for the composition of two $n$-jets is more complicated than for addition and multiplication. It can be derived from the chain rule,

$$
j^{n}(f \circ g)_{i}=\sum_{j=0}^{i} f^{(j)}(g(u)) M_{i j}\left(g^{(1)}(u), \ldots, g^{(i)}(u)\right) .
$$

The formulas for $M$ can be found in [32]. The matrix $M\left(\beta_{1}, \ldots, \beta_{n}\right)$ has the following properties: It is lower triangular, and its entries are polynomials in $\beta_{1}, \ldots, \beta_{n}$. The variable $\beta_{i}$ does not appear in $M_{i j}(\beta)$ for $j>i$. Also, $M_{0 i}(\beta)=$ $M_{i 0}(\beta)=\delta_{i 0}, M_{i 1}(\beta)=\beta_{i}$ for $i \geq 1$, and $M_{i i}(\beta)=\beta_{1}^{i}[21,22]$. Note that right composition with $j^{n} g$ is a linear function on $j^{n} f$, but that left composition with $j^{n} f$ is not a linear function on $j^{n} g$, since $M$ is, in general, polynomial in the derivatives of $g$.

The identity with respect to $n$-jet composition is the $n$-jet of the identity map:

$$
j^{n} I=(0,1,0, \ldots, 0)=I .
$$

It satisfies the following equation:

$$
j^{n} f \circ j^{n} I=j^{n}(f \circ I)=j^{n} f=j^{n}(I \circ f)=j^{n} I \circ j^{n} f .
$$

Note carefully the difference between composition $\left({ }^{\circ}\right)$ and multiplication $(*)$ and their respective identities, $I$ and 1 . The function $I$ takes $u$ to $u$, whereas the function 1 takes $u$ to 1 .

We define the inverse of the $n$-jet of a function as the $n$-jet of the inverse of the function

$$
j^{n} I=j^{n}\left(f \circ f^{-1}\right)=j^{n} f \circ j^{n}\left(f^{-1}\right) .
$$


We denote the inverse $n$-jet,

$$
\left(j^{n} f\right)^{-1}=j^{n}\left(\digamma^{-1}\right) .
$$

In general, the operations of multiplication and composition do not commute,

$$
\left(j^{n} f \circ j^{n} r\right) * j^{n} g \neq\left(j^{n} f * j^{n} g\right) \circ j^{n} r
$$

since, in general,

$$
(f \circ r) g=f(r(u)) g(u) \neq f(r(u)) g(r(u))=(f g) \circ r .
$$

We can, however, "almost commute" them in the following sense:

$$
(f \circ r) g=f(r(u)) g(u)=f(r(u)) g\left(r^{-1}(r(u))\right)=\left(f g^{\prime}\right) \circ r
$$

where $g^{\prime}=g \circ r^{-1}$. Since this is true of functions in general, it is true for their $n$-jets:

$$
\left(j^{n} f \circ j^{n} r\right) * j^{n} g=\left(j^{n} f * j^{n} g^{\prime}\right) \circ j^{n} r .
$$

Given $n$-jets $\alpha$ and $\beta$, there exists an $\alpha^{\prime}$ so that right composition with $\beta$ followed by right multiplication by $\alpha$ is equal to right multiplication by $\alpha^{\prime}$ followed by right composition with $\beta$ :

$$
(\gamma \circ \beta) * \alpha=\left(\gamma * \alpha^{\prime}\right) \circ \beta
$$

for all $\gamma$ in $J^{n}(F)$.

Finally, we introduce the family of linear transformations on $J^{n}(F)$, that is, all functions $L: J^{n}(F) \rightarrow J^{n}(F)$ that can be represented as

$$
\left(L j^{n} f\right)_{i}=\sum_{j=0}^{n} L_{i j} f^{(j)}
$$

Note that left and right multiplication and right composition are all linear transformations, whereas addition and left composition are not. Specifically, multiplication by $j^{n} f$ can be represented by a matrix that we denote $j^{n} f *$ :

$$
j^{n} f * j^{n} g=\left(j^{n} f *\right) j^{n} g
$$

By denoting the matrix in this way, any $n$-jet multiplication $\alpha * \beta$ can also be viewed as the multiplication of $\beta$ by the matrix $\alpha *$. Since we define the results of both operations to be the same, there should be no confusion regarding the dual interpretation. The definition of $\alpha *$ must consequently be

$$
\boldsymbol{\alpha *}=\left[\begin{array}{cccccc}
\alpha_{0} & 0 & 0 & \cdots & 0 & 0 \\
\alpha_{1} & \alpha_{0} & 0 & \cdots & 0 & 0 \\
\alpha_{2} & 2 \alpha_{1} & \alpha_{0} & \cdots & 0 & 0 \\
\vdots & \vdots & \vdots & \cdots & \vdots & \vdots \\
\left(\begin{array}{c}
n-1 \\
0
\end{array}\right) \alpha_{n-1} & \left(\begin{array}{c}
n-1 \\
1
\end{array}\right) \alpha_{n-2} & \left(\begin{array}{c}
n-1 \\
2
\end{array}\right) \alpha_{n-3} & \cdots & \left(\begin{array}{c}
n-1 \\
n-1
\end{array}\right) \alpha_{0} & 0 \\
\left(\begin{array}{c}
n \\
0
\end{array}\right) \alpha_{n} & \left(\begin{array}{c}
n \\
1
\end{array}\right) \alpha_{n-1} & \left(\begin{array}{c}
n \\
2
\end{array}\right) \alpha_{n-2} & \cdots & \left(\begin{array}{c}
n \\
n-1
\end{array}\right) \alpha_{1}\left(\begin{array}{l}
n \\
n
\end{array}\right) \alpha_{0}
\end{array}\right] .
$$


Right composition can be represented by a matrix that we denote $\left(\circ j^{n} g\right)$ or $M\left(j^{n} g\right)$ :

$$
j^{n} f \circ j^{n} g=\left(\circ j^{n} g\right) j^{n} f=M\left(j^{n} g\right) j^{n} f
$$

where

$$
\circ \beta=M(\beta)=\left[\begin{array}{cccccc}
1 & 0 & 0 & \cdots & 0 & 0 \\
0 & \beta_{1} & 0 & \cdots & 0 & 0 \\
0 & \beta_{2} & \beta_{1}^{2} & \cdots & 0 & 0 \\
0 & \vdots & \vdots & \cdots & \vdots & \vdots \\
0 & \beta_{n-1} & \cdots & \cdots & \beta_{1}^{n-1} & 0 \\
0 & \beta_{n} & \cdots & \cdots & \cdots & \beta_{1}^{n}
\end{array}\right]
$$

for $\beta \in J^{n}(F)$. The matrix $M(\beta)$ given above in (58) is used in Section 9 to express Beta-constraints or geometric continuity. Note also that, by inverting the matrices for multiplication and composition, one can compute the reciprocal and inverses of an $n$-jet, respectively. Since both $M\left(j^{n} f\right)$ and $j^{n} f *$ are lower triangular matrices, their determinants are the products of their diagonals:

$$
\begin{gathered}
\operatorname{det}\left(M\left(j^{n} f\right)\right)=\left(f^{(1)}\right)^{n(n+1) / 2} \\
\operatorname{det}\left(j^{n} f *\right)=\left(f^{(0)}\right)^{n} .
\end{gathered}
$$

Thus, $j^{n} f$ has an inverse if and only if $f^{(1)} \neq 0$; moreover, $j^{n} f$ has a reciprocal if and only if $f^{(0)} \neq 0$.

The following generalization of the matrix for right composition will be useful with regard to Frenet frame continuity. Let $M$ be a lower triangular $(n+1) \times$ $(n+1)$ matrix with the following restrictions $[15,22,23]: M_{i 0}=M_{0 i}=\delta_{i 0}$ and $M_{i i}=M_{11}^{i}>0$. Thus, the diagonal is the same as that for $M(\beta)$, but the $n(n-1) / 2$ subdiagonal entries are entirely arbitrary. (In the case of $M(\beta)$, the subdiagonal entries were polynomial functions of $\beta_{1} \cdots \beta_{n}$.) A matrix of this form is called a Frenet frame matrix. Notationally, $M$ and $N$ will be used to denote Frenet frame matrices, while $M(\beta)$ will be used to denote the special case when the Frenet frame matrix is also the matrix for right composition (i.e., the matrix used for Beta-constraints).

As in the case for composition, multiplication by $M$ and multiplication by an $n$-jet can almost be commuted. That is, given a Frenet frame matrix $N$ and an $n$-jet $\beta$ there exist an $n$-jet $\alpha$ and a Frenet frame matrix $M$ so that

$$
\alpha * M=N \beta * \text {. }
$$

The above expression might appear to be missing its right-hand operands. However, when $\alpha *, M, N$, and $\beta *$ are interpreted as matrices the expression is meaningful. First note that

$$
(\alpha * M)_{i 0}=\sum_{j=0}^{n} \alpha *_{i j} M_{j 0}=\sum_{j=0}^{n} \alpha_{i-j}\left(\begin{array}{l}
i \\
j
\end{array}\right) M_{j 0}=\alpha_{i} .
$$

Thus, we must have

$$
\alpha_{i}=(N \beta *)_{i 0} .
$$


And, in particular, we must have

$$
\alpha_{0}=\beta_{0}
$$

With $\alpha$ specified, we must have

$$
M=\frac{1}{\alpha *} N \beta * .
$$

It remains to be shown that $M$ is a Frenet frame matrix. The diagonal elements are

$$
M_{i i}=\left(\frac{1}{\alpha *} N \beta *\right)_{i i}=\left(\frac{1}{\alpha *}\right)_{i i} N_{i i}(\beta *)_{i i} .
$$

Since $(1 / \alpha)_{0}=1 /\left(\alpha_{0}\right)=1 /\left(\beta_{0}\right)$, we have

$$
M_{i i}=\left(\frac{1}{\alpha}\right)_{0} N_{i i} \beta_{0}=N_{i i}=N_{11}^{i} \text {. }
$$

Thus, the diagonal elements are the powers of $N_{11}$. Also,

$$
M_{i 0}=\left(\frac{1}{\alpha *} N \beta *\right)_{i 0}=\sum_{j=0}^{n}\left(\frac{1}{\alpha}\right)_{i-j}\left(\begin{array}{l}
i \\
j
\end{array}\right)(N \beta *)_{j 0}=\sum_{j=0}^{n}\left(\frac{1}{\alpha}\right)_{i-j}\left(\begin{array}{l}
i \\
j
\end{array}\right) \alpha_{j} .
$$

By the definition of $1 / \alpha$, we have

$$
M_{i 0}=\delta_{i 0}
$$

Note that, when we commute the operations of multiplication by an $n$-jet and multiplication by a Frenet frame matrix, both the matrix and the $n$-jet are potentially changed.

So far we have only considered $n$-jets for scalar-valued functions. If we are careful not to attempt to take the reciprocal or inverse of a vector-valued function, then there is no reason that we cannot extend $n$-jets to vector-valued functions.

\section{RATIONAL FRENET FRIAME CONTINUITY}

In [16] it is shown that a curve $\mathbf{q}(u)$ is $F^{n}$ at $t$ if and only if there exists a Frenet frame matrix $M(t)$ such that

$$
\lim _{u \rightarrow t^{+}} j^{n} \mathbf{q}(u)=\lim _{u \rightarrow t^{-}} M(t) j^{n} \mathbf{q}(u) .
$$

A matrix, such as $M(t)$, that relates the derivatives on one side of a parameter value to the derivatives or the other side of the parameter value is called a connection matrix. Thus, a curve is Frenet frame continuous if and only if it has a Frenet frame connection matrix. Using this, we can derive a set of linear componentwise constraints on $\mathbf{Q}$ that are equivalent to $\mathbf{q} \in F^{n}$.

Theorem 1. Rational Frenet frame continuity. Let $\mathbf{q}:(a, b) \rightarrow \mathbb{R}^{d}$, $\mathbf{q}=\left(Q_{1} / Q_{d+1}, \ldots, Q_{d} / Q_{d+1}\right)$, be a regular curve. Suppose that $Q_{d+1}(u) \neq 0$ for all $u \in(a, b)$ and that the one-sided limits of $\mathbf{Q}^{(i)}(u)$ exist for $i=0, \ldots, n$. Then $\mathbf{q}(u)$ is $F^{n}$ if and only if there exist $n$-jets $\alpha(t)$ and a Frenet frame connection matrix 
$M(t)$ with $M_{11}(t)>0$ such that the associated homogeneous curve $\mathbf{Q}: \mathbb{R} \rightarrow \mathbb{R}^{d+1}$ defined by $\mathbf{Q}=\left(Q_{1}, \ldots, Q_{d}, Q_{d+1}\right)$ satisfies

$$
\lim _{u \rightarrow t^{+}} j^{n} \mathbf{Q}(u)=\alpha(t) * M(t)\left(\lim _{u \rightarrow t^{-}} j^{n} \mathbf{Q}(u)\right) .
$$

Note that in this proof there are two Frenet frame continuity connection matrices $M(t)$ and $M^{\prime}(t)$ associated with $\mathbf{Q}(u)$ and $\mathbf{q}(u)$, respectively. These matrices are obtained from one another by commuting with $n$-jets and thus might not be the same. In the special case that the Frenet frame connection matrix is a matrix obtained from right composition, however, commuting will leave $M(t)=M^{\prime}(t)$.

Proof. First, assume that $\mathbf{q}(u)$ is $F^{n}$; by $(70)$, there exists a Frenet frame connection matrix $M^{\prime}(t)$ with $M_{11}^{\prime}(t)>0$ so that

$$
\lim _{u \rightarrow t^{+}} j^{n} \mathbf{q}(u)=M^{\prime}(t) \lim _{u \rightarrow t^{-}} j^{n} \mathbf{q}(u) .
$$

Since $\mathbf{q}(u)=\mathbf{Q}(u) / Q_{d+1}(u)$,

$$
\lim _{u \rightarrow t^{+}} j^{n}\left(\frac{\mathbf{Q}}{\mathbf{Q}_{d+1}}\right)(u)=M^{\prime}(t) \lim _{u \rightarrow t^{-}} j^{n}\left(\frac{\mathbf{Q}}{\mathbf{Q}_{d+1}}\right)(u) .
$$

Applying the definition of $n$-jet multiplication and allowing the factors to proceed to their limits independently yields

$$
\lim _{u \rightarrow t^{+}} \frac{1}{j^{n} Q_{d+1}(u)} * \lim _{u \rightarrow t^{+}} j^{n} \mathbf{Q}(u)=M^{\prime} \lim _{u \rightarrow t^{-}} \frac{1}{j^{n} Q_{d+1}(u)} * \lim _{u \rightarrow t^{-}} j^{n} \mathbf{Q}(u) .
$$

If we denote $\lim _{u \rightarrow t^{+}} j^{n} Q_{d+1}(u)$ and $\lim _{u \rightarrow t^{-}} 1 / j^{n} Q_{d+1}(u)$ by $\phi(t)$ and $\psi(t)$, respectively, we have

$$
\lim _{u \rightarrow t^{+}} j^{n} \mathbf{Q}(u)=\phi^{-1}(t) * M^{\prime}(t)\left(\psi(t) * \lim _{u \rightarrow t^{-}} \mathbf{Q}(u)\right) .
$$

By eq. (61), there exist some $n$-jet $\gamma(t)$ and Frenet frame connection matrix $M(t)$ so that $\gamma(t) * M(t)=M^{\prime}(t) \psi(t) *$ and $M_{11}(t)>0$, so that

$$
\lim _{u \rightarrow t^{+}} j^{n} \mathbf{Q}(u)=\phi^{-1}(t) * \gamma(t) * M(t)\left(\lim _{u \rightarrow t^{-}} \mathbf{Q}(u)\right) .
$$

If we denote the product $\phi^{-1}(t) * \gamma(t)$ by $\alpha(t)$, then we have arrived at eq. (71).

Let us proceed the other way: Assume that eq. (71) holds for some $\alpha(t)$ and Frenet frame connection matrix $M(t)$ with $M_{11}(t)>0$, and then prove that $q$ is $F^{n}$. If we write $Q_{d+1}(u) \mathbf{q}(u)$ for $\mathbf{Q}(u)$ in eq. (71), where $\mathbf{q}(u)$ is meant to be the embedding of $\mathbf{q}(u)$ in $\mathbb{R}^{d+1}$ (i.e., $(\mathbf{q}(u), 1)$ ), then we have

$$
\lim _{u \rightarrow t^{+}} j^{n} Q_{d+1}(u) * j^{n} \mathbf{q}(u)=\alpha(t) * M(t)\left(\lim _{u \rightarrow t^{-}} j^{n} Q_{d+1} * j^{n} \mathbf{q}(u)\right)
$$


If we denote $\lim _{u \rightarrow t^{+} j} Q_{d+1}$ by $\gamma(t)$ and $\lim _{u \rightarrow t^{-}} j^{n} Q_{d+1}$ by $\psi(t)$ and let the factors proceed to their limits independently, then

$$
\gamma(t) * \lim _{u \rightarrow t^{+}} j^{n} \mathbf{q}(u)=\alpha(t) * M(t)\left(\psi(t) * \lim _{u \rightarrow t^{-}} j^{n} \mathbf{q}(u)\right) .
$$

Multiplying both sides by $1 / \gamma(t)$ and substituting $\psi^{\prime}(t) * M^{\prime}(t)$ for $M(t) \psi *(t)$ gives:

$$
\lim _{u \rightarrow t^{+}} j^{n} \mathbf{q}(u)=\frac{1}{\gamma(t)} * \alpha(t) * \psi^{\prime}(t) * M^{\prime}(t) \lim _{u \rightarrow t^{-}} j^{n} \mathbf{q}(u)
$$

The $d+1$ st component of $\mathbf{q}(u)$ is the constant function 1 , so the above equation implies that

$$
j^{n} 1=\frac{1}{\gamma(t)} * \alpha(t) * \psi^{\prime}(t) * M^{\prime}(t) j^{n} 1
$$

Since $M^{\prime}(t) j^{n} 1=j^{n} 1$, we have

$$
j^{n} 1=\frac{1}{\gamma(t)} * \alpha(t) * \psi^{\prime}(t)
$$

Thus,

$$
\lim _{u \rightarrow t^{+}} j^{n} \mathbf{q}(u)=M^{\prime}(t) \lim _{u \rightarrow t^{-}} j^{n} \mathbf{q}(u),
$$

and thus, $\mathbf{q}(u)$ is Frenet frame continuous.

\section{RATIONAL GEOMETRIC CONTINUITY}

Recall that a curve $\mathbf{q}$ is geometrically continuous $\left(G^{n}\right)$ if there exists a reparametrization $r(u)$ such that $\mathbf{q}(r(u))$ is regular and parametrically continuous. Geometric continuity can be posed as a set of constraints on the components of q. Barsky and DeRose have shown $[5-7,14]$ that a regular curve $\mathbf{q}$ is $G^{n}$ if and only if there exist scalars $\beta_{i}(t), i=1, \ldots, n$ with $\beta_{1}(t)>0$ such that

$$
\lim _{u \rightarrow t^{+}} j^{n} \mathbf{q}(u)=\lim _{u \rightarrow t^{-}} M(\beta(t)) j^{n} \mathbf{q}(u)
$$

where $M(\beta(t))$ is the matrix defined by eq. (44). This equation is simply the Beta-constraints written in matrix notation. The Beta-constraints can be found in the Appendix. Using this, we can derive a set of linear componentwise constraints on $\mathbf{Q}$ that are equivalent to $\mathbf{q} \in G^{n}$.

CorollaRy 1. Rational geometric continuity. Let q: $(a, b) \rightarrow \mathbb{R}^{d}$, $\mathbf{q}=\left(Q_{1} / Q_{d+1}, \ldots, Q_{d} / Q_{d+1}\right)$, be a regular curve. Suppose that $Q_{d+1}(u) \neq 0$ for all $u \in(a, b)$ and that the one-sided limits of $\mathbf{Q}^{(i)}(u)$ exist for $i=0, \ldots, n$. Then $\mathbf{q}(u)$ is $G^{n}$ if and only if there exist $n$-jets $\alpha(t)$ and $\beta(t)$, with $\beta_{0}(t)=t$ and $\beta_{1}(t)>0$, such that the associated homogeneous curve $\mathbf{Q}: \mathbb{R} \rightarrow \mathbb{R}^{d+1}$ defined by $\mathbf{Q}=\left(Q_{1}, \ldots\right.$, $\left.Q_{d}, Q_{d+1}\right)$ satisfies

$$
\lim _{u \rightarrow t^{+}} j^{n} \mathbf{Q}(u)=\alpha(t) * M(\beta(t))\left(\lim _{u \rightarrow t^{-}} j^{n} \mathbf{Q}(u)\right)
$$


Note that if $Q_{d+1}(u) \neq 0$ then any $\alpha_{0}(t)$ satisfying (84) will be nonzero for all $t$. The continuity constraints are given explicitly in the Appendix.

Proof. If $M(\beta(t))$ is substituted for $M^{\prime}(t)$ and $M(t)$ in Theorem 1 , then by eq. (53) the commutations in eqs. (76) and (79) can take place with $M^{\prime}(t)=M(t)$.

\section{RATIONAL PARAMETRIC CONTINUITY}

Corollary 2. Rational parametric continuity. Let $\mathbf{Q}(u)$ be a homogeneous curve whose one-sided derivatives exist everywhere on an interval $(a, b)$ and whose value at every parameter is equal to the limit from the left or the limit from the right. Further, assume that $Q_{d+1}(u)$ is never zero. The projection of $\mathbf{Q}(u)$ is $C^{n}$ on $(a, b)$ if and only if there exists an $n$-jet $\alpha(t):(a, b) \rightarrow J^{n}(F)$ so that

$$
\lim _{u \rightarrow t^{+}} j^{n} \mathbf{Q}(u)=\alpha(t) * \lim _{u \rightarrow t^{-}} j^{n} \mathbf{Q}(u) \text {. }
$$

Proof. If $M(t)$ is set to the identity in Theorem 1 , the corollary follows immediately.

Just as a matrix that relates the derivatives on one side of a parameter value to the derivatives on the other side of the parameter value is called a connection matrix, an $n$-jet that relates the derivatives on one side of a parameter value to the derivatives on the other side of the parameter value is called a connection $n$-jet. The above theorem states that a homogeneous curve $\mathbf{Q}$ projects to a $C^{n}$ curve $\mathbf{q}$ if and only if $\mathbf{Q}$ has a connection $n$-jet.

\section{SMOOTHING MULTIPLICATION}

We have seen that it is possible to divide each component of a function by a common function to obtain a function that is $G^{n}$ even if neither the original function nor the divisor was $C^{n}$. One might ask a similar question about multiplication instead of division. That is, what restrictions must be placed on $g(u)$ and $\mathbf{q}(u)$ so that $\mathbf{q}(u) g(u)$ is $G^{n}$ ? We have depicted this in Figure 6.

CoRollary 3. Multiplying to make curves geometrically continuous. A regular curve $\mathbf{q}(u) g(u)$ will be $G^{n}$ on $(a, b)$ if and only if there exist $n$-jets $\alpha(t)$ and $\beta(t)$ with $\beta_{1}(t)>0$ for all $t$ such that

$$
\lim _{u \rightarrow t^{+}} j^{n} \mathbf{q}(u)=\alpha(t) *\left(M(\beta(t)) \lim _{u \rightarrow t^{-}} j^{n} \mathbf{q}(u)\right)
$$

and

$$
\lim _{u \rightarrow \iota^{+}} j^{n} g(u)=\frac{1}{\alpha(t)} *\left(M(\beta(t)) \lim _{u \rightarrow t^{-}} j^{n} g(u)\right) .
$$

Proof. The curve $\mathbf{q}(u) g(u)$ in $\mathbb{R}^{d}$ can be viewed as the projection from $\mathbb{R}^{d+1}$ to $\mathbb{R}^{d}$ of the curve $(\mathbf{q}(u), 1 / g(u))$. By Corollary $1, \mathbf{q}(u) g(u)$ will be $G^{n}$ if and only if there exist $n$-jets $\alpha(t), \beta(t):(a, b) \rightarrow J^{n}(F)$ such that

$$
\lim _{u \rightarrow t^{+}} j^{n}\left(\mathbf{q}(u), \frac{1}{g(u)}\right)=\alpha(t) *\left(M(\beta(t)) \lim _{u \rightarrow t^{-}} j^{n}\left(\mathbf{q}(u), \frac{1}{g(u)}\right)\right) .
$$


Fig. 6. For $\mathbf{q}(u) g(u)$ to be $G^{n}$, the connection $n$-jet for $q$ must be the reciprocal of the connection $n$-jet for $g$.

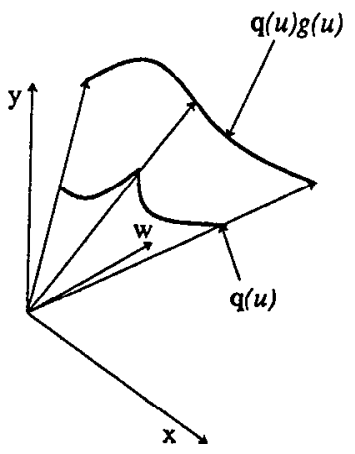

This is equivalent to

$$
\lim _{u \rightarrow t^{+}} j^{n} \mathbf{q}(u)=\alpha(t) *\left(M(\beta(t)) \lim _{u \rightarrow t^{-}} j^{n} \mathbf{q}(u)\right)
$$

and

$$
\lim _{u \rightarrow t^{+}} j^{n}\left(\frac{1}{g(u)}\right)=\alpha(t) * M(\beta(t)) \lim _{u \rightarrow t^{-}} j^{n}\left(\frac{1}{g(u)}\right) .
$$

Equation (90) can be rewritten as

$$
\lim _{u \rightarrow t^{+}} j^{n}\left(\frac{1}{g(u)}\right)=\alpha(t) * \lim _{u \rightarrow t^{-}} j^{n}\left(\frac{1}{g(u)} \circ \beta(t)\right) .
$$

Inverting the $n$-jets gives

$$
\lim _{u \rightarrow t^{+}} j^{n} g(u)=\frac{1}{\alpha(t)} *\left(\lim _{u \rightarrow t^{-}} j^{n} g(u) \circ \beta(t)\right)
$$

or

$$
\lim _{u \rightarrow t^{+}} j^{n} g(u)=\frac{1}{\alpha(t)} *\left(M(\beta(t)) \lim _{u \rightarrow t^{-}} j^{n} g(u)\right) .
$$

CoRollary 4. Multiplying to make curves parametrically continuous. The homogeneous curve $\mathbf{q}(u) g(u)$ will be $C^{n}$ on an interval $(a, b)$ if and only if $\mathbf{q}(u)$ has a connection $n$-jet on $(a, b)$ and that connection $n$-jet is the reciprocal of the connection $n$-jet for $g(u)$.

Proof. This follows directly if we set $\beta(t)=J^{n} I$ in Corollary 3 .

Remark. A result similar to Corollaries 3 and 4 has not yet been shown for Frenet frame continuity.

\section{DISCUSSION}

In Figure 7, we have depicted rational geometric continuity of the first degree. We have depicted a curve $\mathbf{R}$ that is composed of $\mathbf{P}(u)$ for $u \leq t$ and $\mathbf{Q}(u)$ for $u>t$. We concern ourselves with the constraints imposed on $\mathbf{Q}^{\prime}(t)$ if $\mathbf{P}$ is ACM Transactions on Graphics, Vol. 8, No. 4, October 1989. 


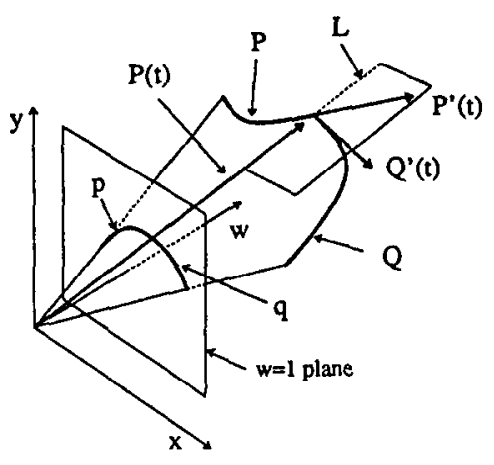

Fig. 7. Rational geometric continuity of the first degree.

known. For simplicity, assume that $\mathbf{R} \in C^{0}$. If we require that $\mathbf{R}$ be $C^{1}$, then $\mathbf{Q}^{\prime}(t)$ is specified exactly by $\mathbf{P}^{\prime}(t)$. If we require that $\mathbf{r}$ be $C^{1}$, then $\mathbf{Q}^{\prime}(t)$ is constrained as shown in Figure 5. If we require that $\mathbf{R}$ be $G^{1}$, then $\mathbf{Q}^{\prime}(t)$ must be a positive scalar multiple of $\mathbf{P}^{\prime}(t)$. If we only require that $\mathbf{r}$ be $G^{1}$, however, then $\mathbf{Q}^{\prime}(t)$ is only required to lie in the half plane $L$ spanned by $\mathbf{P}(t)$ and positive scalar multiples of $\mathbf{P}^{\prime}(t)$.

In general, if $\mathbf{P}$ is known then the derivative $\mathbf{Q}^{(i)}(t)$ will be determined by the set of $\alpha \mathrm{s}$ and $\beta \mathrm{s}$ :

$$
\begin{aligned}
\mathbf{Q}^{(i)}(t)= & \alpha_{i} \mathbf{P}(t)+\alpha_{0} \beta_{i} \mathbf{P}^{\prime}(t) \\
& + \text { other terms involving } \alpha_{j} \text { and } \beta_{j} \text { where } j \neq i .
\end{aligned}
$$

We would like to know if $\alpha_{i}$ gives us a degree of freedom that we did not already have through $\beta_{i}$. If $\mathbf{P}(t)$ and $\mathbf{P}^{\prime}(t)$ are linearly independent, as we pictured them to be in Figure 7 , then by varying $\alpha_{i}$ we can specify $\mathbf{Q}^{(i)}(t)$ in a way not possible with $\beta_{i}$. By the following argument, if the curve $\mathbf{p}$ is regular then $\mathbf{P}(t)$ and $\mathbf{P}^{\prime}(t)$ will be linearly independent.

Suppose that $\mathbf{p}(u)$ is regular. Then

$$
\mathbf{p}^{\prime}(u) \neq 0 .
$$

At least one component, $p_{i}^{\prime}(u)$, of $\mathbf{p}^{\prime}(u)$ cannot be zero:

$$
p_{i}^{\prime}(u)=\frac{P_{i}^{\prime}(u) P_{d+1}(u)-P_{i}(u) P_{d+1}^{\prime}(u)}{P_{d+1}^{2}(u)} \neq 0 .
$$

Thus,

$$
P_{i}^{\prime}(u) P_{d+1}(u)-P_{i}(u) P_{d+1}^{\prime}(u) \neq 0 .
$$

The left-hand side of (97) is the first component of $\mathbf{A} \times \mathbf{B}$ where $\mathbf{A}=\left(0, P_{i}(u)\right.$, $\left.P_{d+1}(u)\right)$ and $\mathbf{B}=\left(0, P_{i}^{\prime}(u), P_{d+1}^{\prime}(u)\right)$. Therefore, $\mathbf{A} \times \mathbf{B} \neq 0$, and $\mathbf{A}$ and $\mathbf{B}$ are linearly independent. If a subset of the components of two vectors are linearly independent, then the vectors themselves must also be; thus, $\mathbf{P}(u)$ and $\mathbf{P}^{\prime}(u)$ are linearly independent. Thus, we are guaranteed that each rational geometric continuity constraint will give us two independent degrees of freedom. 


\section{CONCLUSIONS}

In this paper we have shown that the three common measures of conlinuilyparametric, geometric, and Frenet frame continuity-are too restrictive to be applied to the homogeneous curve associated with a rational curve. By investigating the representation of a circle by piecewise rational curves, we have established the necessity of using rational curves whose homogeneous representation is not smooth. We have derived the constraints on the homogeneous curve that are exactly equivalent to the parametric, geometric, and Frenet frame continuity of the rational curve. For every degree of continuity, the rational continuity constraints contain a degree of freedom not present in the corresponding continuity constraints for projected curves. The construction of spline curves based on these rational continuity constraints is investigated in $[35,36]$.

\section{APPENDIX}

\section{Rational Parametric Continuity Constraints}

The projection of $\mathbf{Q}(u)$ will be $C^{n}$ at parameter value $t$ if and only if there exist $\alpha_{i}$ so that

$$
\lim _{u \rightarrow t^{+}} \mathbf{Q}^{(i)}(u)=\lim _{u \rightarrow t^{-}} \sum_{j=0}^{i}\left(\begin{array}{c}
i \\
j
\end{array}\right) \alpha_{-j} \mathbf{Q}^{(j)}(u) \quad i=0, \ldots, n .
$$

The first few constraints are

$$
\begin{gathered}
\lim _{u \rightarrow t^{+}} \mathbf{Q}^{(0)}(u)=\lim _{u \rightarrow t^{-}} \alpha_{0} \mathbf{Q}^{(0)}(u), \\
\lim _{u \rightarrow t^{+}} \mathbf{Q}^{(1)}(u)=\lim _{u \rightarrow t^{-}}\left[\alpha_{1} \mathbf{Q}^{(0)}(u)+\alpha_{0} \mathbf{Q}^{(1)}(u)\right], \\
\lim _{u \rightarrow t^{+}} \mathbf{Q}^{(2)}(u)=\lim _{u \rightarrow t^{-}}\left[\alpha_{2} \mathbf{Q}^{(0)}(u)+2 \alpha_{1} \mathbf{Q}^{(1)}(u)+\alpha_{0} \mathbf{Q}^{(2)}(u)\right], \\
\lim _{u \rightarrow t^{+}} \mathbf{Q}^{(3)}(u)=\lim _{u \rightarrow t^{-}}\left[\alpha_{3} \mathbf{Q}^{(0)}(u)+3 \alpha_{2} \mathbf{Q}^{(1)}(u)+3 \alpha_{1} \mathbf{Q}^{(2)}(u)+\alpha_{0} \mathbf{Q}^{(3)}(u)\right], \\
\lim _{u \rightarrow t^{+}} \mathbf{Q}^{(4)}(u)=\lim _{u \rightarrow t^{-}}\left[\alpha_{4} \mathbf{Q}^{(0)}(u)+4 \alpha_{3} \mathbf{Q}^{(1)}(u)\right. \\
\left.+6 \alpha_{2} \mathbf{Q}^{(2)}(u)+4 \alpha_{1} \mathbf{Q}^{(3)}(u)+\alpha_{0} \mathbf{Q}^{(4)}(u)\right] .
\end{gathered}
$$

Note that if $\alpha_{0}=1$ and $\alpha_{i}=0$ for $i \geq 1$ then rational parametric continuity reduces to simple parametric continuity.

\section{Geometric Continuity Constraints}

A curve $\mathbf{q}(u)$ will be $G^{n}$ at parameter value $t$ if and only if there exist $\beta_{i}$ such that

$$
\lim _{u \rightarrow t^{+}} \mathbf{q}^{(i)}(u)=\lim _{u \rightarrow t^{-}} \sum_{j=0}^{i} M_{i j}(\beta) \mathbf{q}^{(j)}(u) \quad i=0, \ldots, n .
$$

These constraints are called the Beta-constraints. The first few constraints are

$$
\begin{gathered}
\lim _{u \rightarrow t^{+}} \mathbf{q}^{(0)}(u)=\lim _{u \rightarrow t^{-}} \mathbf{q}^{(0)}(u), \\
\lim _{u \rightarrow t^{+}} \mathbf{q}^{(1)}(u)=\lim _{u \rightarrow t^{-}} \beta_{1} \mathbf{q}^{(1)}(u),
\end{gathered}
$$




$$
\begin{gathered}
\lim _{u \rightarrow t^{+}} \mathbf{q}^{(2)}(u)=\lim _{u \rightarrow t^{-}}\left[\beta_{1}^{2} \mathbf{q}^{(2)}(u)+\beta_{2} \mathbf{q}^{(1)}(u)\right] \\
\lim _{u \rightarrow t^{+}} \mathbf{q}^{(3)}(u)=\lim _{u \rightarrow t^{-}}\left[\beta_{1}^{3} \mathbf{q}^{(3)}(u)+3 \beta_{1} \beta_{2} \mathbf{q}^{(2)}(u)+\beta_{3} \mathbf{q}^{(1)}(u)\right] \\
\lim _{u \rightarrow t^{+}} \mathbf{q}^{(4)}(u)=\lim _{u \rightarrow t^{-}}\left[\beta_{1}^{4} \mathbf{q}^{(4)}(u)+6 \beta_{1}^{2} \beta_{2} \mathbf{q}^{(3)}(u)\right. \\
\left.+\left(4 \beta_{1} \beta_{3}+3 \beta_{2}^{2}\right) \mathbf{q}^{(2)}(u)+\beta_{4} \mathbf{q}^{(1)}(u)\right]
\end{gathered}
$$

\section{Rational Geometric Continuity Constraints}

The projection of $\mathbf{Q}(u)$ will be $G^{n}$ if and only if there exist $\alpha_{i}$ and $\beta_{i}$ such that

$$
\lim _{u \rightarrow t^{+}} \mathbf{Q}^{(i)}(u)=\sum_{j=0}^{i} \lim _{u \rightarrow t^{-}}\left(\begin{array}{c}
i \\
j
\end{array}\right) \alpha_{i-j} \sum_{k=0}^{j} M_{j k}(\beta) \mathbf{Q}^{(k)}(u) \quad i=0, \ldots, n .
$$

The first few constraints are

$$
\begin{gathered}
\lim _{u \rightarrow t^{+}} \mathbf{Q}^{(0)}(u)=\lim _{u \rightarrow t^{-}} \alpha_{0} \mathbf{Q}^{(0)}(u) \\
\lim _{u \rightarrow t^{+}} \mathbf{Q}^{(1)}(u)=\lim _{u \rightarrow t^{-}}\left[\alpha_{1} \mathbf{Q}^{(0)}(u)+\alpha_{0} \beta_{1} \mathbf{Q}^{(1)}(u)\right] \\
\lim _{u \rightarrow t^{+}} \mathbf{Q}^{(2)}(u)=\lim _{u \rightarrow t^{-}}\left[\alpha_{2} \mathbf{Q}^{(0)}(u)+\left(\alpha_{0} \beta_{2}+2 \alpha_{1} \beta_{1}\right) \mathbf{Q}^{(1)}(u)+\alpha_{0} \beta_{1}^{2} \mathbf{Q}^{(2)}(u)\right] \\
\lim _{u \rightarrow t^{+}} \mathbf{Q}^{(3)}(u)=\lim _{u \rightarrow t^{-}}\left[\alpha_{3} \mathbf{Q}^{(0)}(u)+\left(\alpha_{0} \beta_{3}+3 \alpha_{1} \beta_{2}+3 \alpha_{2} \beta_{1}\right) \mathbf{Q}^{(1)}(u)\right. \\
\left.+3\left(\alpha_{0} \beta_{1} \beta_{2}+\alpha_{1} \beta_{1}^{2}\right) \mathbf{Q}^{(2)}(u)+\alpha_{0} \beta_{1}^{3} \mathbf{Q}^{(3)}(u)\right] \\
\lim _{u \rightarrow t^{+}} \mathbf{Q}^{(4)}(u)=\lim _{u \rightarrow t^{-}}\left[\alpha_{4} \mathbf{Q}^{(0)}(u)+\left(\alpha_{0} \beta_{4}+4 \alpha_{1} \beta_{3}+6 \alpha_{2} \beta_{2}+4 \alpha_{3} \beta_{1}\right) \mathbf{Q}^{(1)}(u)\right. \\
+\left(4 \alpha_{0} \beta_{1} \beta_{3}+3 \alpha_{0} \beta_{2}^{2}+12 \alpha_{1} \beta_{1} \beta_{2}+6 \alpha_{2} \beta_{1}^{2}\right) \mathbf{Q}^{(2)}(u) \\
\left.+\left(6 \alpha_{0} \beta_{1}^{2} \beta_{2}+4 \alpha_{1} \beta_{1}^{3}\right) \mathbf{Q}^{(3)}(u)+\alpha_{0} \beta_{1}^{4} \mathbf{Q}^{(4)}(u)\right]
\end{gathered}
$$

If we let $\alpha_{0}=1$ and $\alpha_{i}=0$ for $i \geq 1$, then the rational geometric continuity constraints reduce to the Beta-constraints. On the other hand, if we let $\beta_{1}=1$ and $\beta_{i}=0$ for $i \geq 2$, then the rational geometric continuity constraints reduce to the rational parametric continuity constraints. Finally, if both $\alpha_{i}$ and $\beta_{i}$ are specified as above, the rational geometric continuity constraints reduce to simple parametric continuity constraints.

\section{REFERENCES}

1. BARSKy, B. A. The Beta-spline: A local representation based on shape parameters and fundamental geometric measures. Ph.D. thesis, Dept. of Computer Science, Univ. of Utah, Salt Lake City, Utah, Dec. 1981.

2. BARSKY, B. A. Introducing the rational Beta-spline. In Proceedings of the 3rd International Conference on Engineering Graphics and Descriptive Geometry, Vol. 1 (Vienna, Austria, July 1116, 1988). American Society for Engineering Education, 1988, pp. 16-27.

3. Bansky, B. A. Computer Graphics and Geometric Modeling Using Beta-splines. Springer-Verlag, Heidelberg, 1988.

4. BARsky, B. A. The rational Beta-spline form for curve and surface representation. Submittcd for publication. 
5. BARSKY, B. A., AND DeRose, T. D. Geometric continuity of parametric curves. Tech. Rep. UCB/CSD 84/205, Computer Science Division, Electrical Engineering and Computer Sciences Dept., Univ. of California, Berkeley, Oct. 1984.

6a. BARSKY, B. A., AND DERose, T. D. Geometric continuity of parametric curves: Three equivalent characterizations. IEEE Comput. Gr. Appl. 9, 6 (Nov. 1989), 60-68.

6b. BARSKY, B. A., AND DEROSE:, T. D. Geometric continuity of parametric curves: Constructions of geometrically continuous splines. IEEE Comput. Gr. Appl. 10, 1 (Jan. 1990), 60-68.

7. BARSKY, B. A., AND DERose, T. D. Geometric continuity of parametric curves: Developing the Beta-constraints. Submitted for publication.

8. Bartels, R. H., BeatTy, J. C., AND Barsky, B. A. An Introduction to Splines for Use in Computer Graphics and Geometric Modeling. Morgan Kaufmann, San Mateo, Calif., 1987.

9. Boenm, W. Curvature continuous curves and surfaces. Comput.-Aided Geom. Des. 2, 4 (Dec. 1985), 313-323.

10. Bоенм, W. Rational geometric splines. Comput.-Aided Geom. Des. 4, 1-2 (July 1987), 67-77.

11. BoenM, W. Smooth curves and surfaces. In Geometric Modeling: Algorithms and New Trends, G. Farin, Ed. Society for Industrial and Applied Mathematics, Philadelphia, Pa., 1987, pp. 175-184.

12. CoHEN, D., AND LEE, T. M. P. Fast drawing of curves for computer display. In Proceedings of the Spring Joint Computer Conference. AFIPS Press, Montvale, N.J., 1969, pp. 297-307.

13. DeRose, A. D. Geometric continuity: A parameterization independent measure of continuity for computer aided geometric design. Ph.D. thesis, Computer Science Div., Dept. of Electrical Engineering and Computer Sciences, Univ. of California, Berkeley, Aug. 1985. (Also available as Tech. Rep. 86-09-04, Dept. of Computer Science, Univ. of Washington, Seattle, 1986; and as Tech. Rep. UCB/CSD 86/25i5, Computer Science Div., Dept. of Electrical Engineering and Computer Sciences, Univ. of California, Berkeley, 1986.)

14. DEROSE, T. D., AND BARSKY, B. A. An intuitive approach to geometric continuity for parametric curves and surfaces. In Proceedings of Graphics Interface '85 (Montreal, May 27-31, 1985). Canadian Information Processing Society, 1985, pp. 343-351. Extended abstract in Proceedings of the International Conference on Computational Geometry and Computer-Aided Design (New Orleans, June 5-8). 1985, pp. 71-75. Revised version published in Computer-Generated ImagesThe State of the Art, N. Magnenat-Thalmann and D. Thalmann, Eds. Springer-Verlag, New York, 1985, pp. 159-175.

15. Dyn, N., Edelman, A., AND Micchelli, C. A. On locally supported basis functions for the representation of geometrically continuous curves. Res. Rep. 12119, IBM Thomas J. Watson Research Center, Yorktown Heights, N.Y., Sept. 1985.

16. Dyn, N., AND Micchelli, C. A. Piecewise polynomial spaces and geometric continuity of curves. Res. Rep. 11390, IBM. Thomas J. Watson Research Center, Yorktown Heights, N.Y., Sept. 1985.

17. FarIN, G. Visually C2 cubic splines. Comput.-Aided Des. 14, 3 (May 1982), 137-139.

18. FARIN, G. Algorithms for rational Bézier curves. Comput.-Aided Des. 15, 2 (Mar., 1983), 73-77.

19. FAux, I. D., AND PratT, M. J. Computational Geometry for Design and Manufacture. Ellis Horwood, Chichester, England, 1979.

20. Fowler, A. H., AND WILSON, C. W. Cubic spline, a curve fitting routine. Corp. Rep. Y-1400 (Rev. I.), Union Carbide 1966.

21. Goldman, R. N., AND BARSK $r$, B. A. $\beta$-continuity and its application to rational Beta-splines. In Proceedings of the 4th Computer Graphics Conference (Smolenice, Czechoslovakia, May, 1518, 1989). (Also Tech. Rep. UCB/CSD 88/442, Computer Science Division, Electrical Engineering and Computer Sciences Dept., Univ. of California, Berkeley, Aug. 1988.)

22. Goldman, R. N., AND BarSKy, B. A. On $\beta$-continuous functions and their application to the construction of geometrically continuous curves and surfaces. In Mathematical Methods in Computer Aided Geometric Design, T. Lyche and L. L. Schumaker, Eds. Academic Press, Boston, 1989, pp. 299-311. (Conference held June 16-22, 1988, in Oslo.)

23. Goldman, R. N., AND Micchislli, C. A. Algebraic aspects of geometric continuity. In Mathematical Methods in Computer Aided Geometric Design, T. Lyche and L. L. Schumaker, Eds. Academic Press, Boston, 1989, pp. 313-332. (Conference held June 16-22, 1988, in Oslo.)

24. Goodman, T. N. T. Properties of Beta-splines. J. Approx. Theory 44, 2 (June 1985), 132-153.

ACM Transactions on Graphics, Vol. 8, No. 4, October 1989. 
25. GoOdman, T. N. T., AND Unsworth, K. Manipulating shape and producing geometric continuity in Beta-spline curves. IEEE Comput. Gr. Appl. 6, 2 (Feb. 1986), 50-56. (Special Issue on Parametric Curves and Surfaces.)

26. Hagen, H. Geometric spline curves. Comput.-Aided Geom. Des. 2, 1-3 (Sept. 1985), 223-227.

27. HAGEN, H. Bézier-curves with curvature and torsion continuity. Rocky Mountain J. Math. 16, 3 (Summer 1986), 629-636.

28. Herron, G. Techniques for visual continuity. In Geometric Modeling: Algorithms and New Trends, G. Farin, Ed. Society for Industrial and Applied Mathematics, Philadelphia, Pa., 1987, pp. 163-174.

29. Hirsch, M. W. Differential Topology. Springer-Verlag, New York, 1976.

30. Hoellig, K. Geometric continuity of spline curves and surfaces. Tech. Rep. 645, Computer Science Dept., Univ. of Wisconsin, Madison, 1986.

31. JoE, B. Rational Beta-spline curves and surfaces and discrete Beta-splines. Tech. Rep. TR8704, Dept. of Computing Science, Univ. of Alberta, Edmonton, Canada, Apr. 1987.

32. KNUTh, D. E. The Art of Computer Programming: Volume 1, Fundamental Algorithms. 2nd ed. Addison-Wesley, Reading, Mass., 1973.

33. Lu, Y.-C. Singularity Theory and an Introduction to Catastrophe Theory. Springer-Verlag, New York, 1976.

34. Manning, J. R. Continuity conditions for spline curves. Comput. J. 17, 2 (May 1974), 181-186.

35. Manocha, D., AND Barsky, B. A. Basis functions for rational continuity. Submitted for publication.

36. MANOCHA, D., AND BARSKY, B. A. Varying the shape parameters of rational continuity. In preparation.

37. Nielson, G. M. Some piecewise polynomial alternatives to splines under tension. In Computer Aided Geometric Design, R. E. Barnhill and R. F. Riesenfeld, Eds. Academic Press, New York, 1974, pp. 209-235.

38. PIEGL, L. Representation of quadric primitives by rational polynomials. Comput.-Aided Geom. Des. 2, 1-3 (Sept. 1985), 151-155.

39. PIEgl, L. The sphere as a rational Bézier surface. Comput.-Aided Geom. Des. 3, 1 (May 1986), 45-52.

40. PIEGL, L. A geometric investigation of the rational Bezzier scheme of computer aided design. Comput. Ind. 7, 5 (Oct. 1986), 401-410.

41. PIEGL, L. Infinite control points-A method for representing surfaces of revolution using boundary data. IEEE Comput. Graph. Appl. 7, 3 (Mar. 1987), 45-55.

42. PIEGL, L. On the use of infinite control points in computer aided geometric design. Comput.Aided Geom. Des. 4, 1-2 (July, 1987), 155-166.

43. Piegl, L., AND Tiller, W. Curve and surface constructions for computer aided design using rational B-splines. Comput.-Aided Des., 19, 9 (Nov. 1987), 485-498.

44. RoBErTs, L. G. Homogeneous matrix representation and manipulation of $N$-dimensional constructs. Comput. Display Rev. (May 1965), 1-16. (Also Doc. MS-1405, Iincoln Lah., MIT, Cambridge, Mass., July 1966.)

45. SABIN, M. A. Parametric splines in tension. Tech. Rep. VTO/MS/160, British Aircraft Corp., Weybridge, Survey, England, July 1970.

46. SpIVAK, M. A Comprehensive Introduction to Differential Geometry, Vol. III. Publish or Perish, Boston, 1973.

47. TILlER, W. Rational B-splines for curve and surface representation. IEEE Comput. Graph. Appl. 3, 6 (Sept. 1983), 61-69.

48. VerSPRILLE, K. J. Computer-aided design applications of the rational B-spline approximation form. Ph.D. thesis, Dept. of Computer and Information Science, Syracuse Univ., Syracuse, N.Y., Feb. 1975.

Received September 1988; accepted January 1989 